\title{
Manejando por Santiago. Explorando el uso de automóviles por parte de habitantes de bajos ingresos desde una óptica de movilidad sustentable*
}

\author{
SEBASTIÁN URETA ICAZA** \\ ** Instituto de Sociología, Pontificia Universidad Católica de Chile.
}

\begin{abstract}
In recent years, existing forms of daily urban mobility and their social and environmental impacts have become issues of debate and public concern. In this article we explore this issue through a specific case study: the analysis of the motives and perceptions that lie behind the acquisition and use of cars by low income residents in Santiago de Chile. Firstly we analyze how the car and the type of urban mobility that is generated are understood in terms of social and urban theory. Secondly, we present some general tendencies of car use in Santiago. Next we look in detail at the principal arguments behind acquisition, use and valuing of the vehicle by a group of low income users in the city. Finally, we offer, based on the analysis, some ideas that can contribute to the design of public policies that incentivize forms of sustainable urban mobility in the city.
\end{abstract}

KEYWORDS: cars, automobility, Santiago de Chile, users, sustainability.

RESUMEN En los últimos años, los regímenes actuales de movilidad urbana cotidiana y sus efectos sociales y medioambientales han sido materia de debate y preocupación pública. En el presente artículo pretendemos explorar el tema a través de un caso de estudio específico: el análisis de los motivos y percepciones detrás de la adquisición y el uso de automóviles por parte de habitantes de bajos ingresos de la ciudad de Santiago, Chile. En primer lugar veremos cómo puede ser entendido el automóvil y el tipo de movilidad urbana que genera desde una óptica de teoría social y urbana. En segundo lugar, presentaremos algunas tendencias generales de uso de automóviles en Santiago. Luego se analizarán en detalle los principales argumentos detrás de la adquisición, uso y valoración del automóvil por parte de un grupo de usuarios de bajos ingresos de la ciudad. Finalmente entregaremos, a partir de los análisis presentados, algunas ideas que pueden contribuir al diseño de políticas públicas que incentiven formas de movilidad urbana sustentables en la ciudad.

PALABRAS CLAVE: automóviles, automovilidad, Santiago de Chile, usuarios, sustentabilidad.

Este artículo forma parte de los resultados del proyecto de investigación "Tecnologías de la comunicación y el transporte en la vida cotidiana de los habitantes de la ciudad de Santiago, Chile", financiado por Fondecyt (proyecto 11060348). Agradezco la colaboración como ayudantes de investigación de Wilson Muñoz, Joan José Richter y Miguel Órdenes.

Recibido el 5 de enero de 2009, aprobado el 12 de junio de 2009.

Correspondencia: Sebastián Ureta Icaza, Instituto de Sociología, Pontificia Universidad Católica de Chile, Vicuña Mackenna 4860, Santiago, Chile. E-mail: sureta@uc.cl 


\section{Introducción}

La movilidad espacial mediante el uso de tecnologías del transporte es central en el modo en que las personas viven los espacios urbanos contemporáneos. Desde el acceso al lugar de trabajo o estudio hasta las formas en las que se utiliza el tiempo libre, el habitar en la ciudad contemporánea está estructurado en torno a crecientes niveles de movimiento entre diversos lugares. Este hecho ha llevado a que incluso se haya comenzado a hablar de la existencia de una "sociedad móvil" (Urry, 2000), en la que los modos tradicionales y estáticos de concebir y usar el espacio urbano (agrupados alrededor de conceptos como hogar, lugar de trabajo, escuelas, barrios, etc.) empiezan a convivir, y en algunos casos a ser reemplazados, por entidades en que la movilidad cumple un rol central (como redes, nodos, flujos, etc.)

Sin embargo, el aumento de la movilidad espacial también ha tenido sus costos. Actualmente existe un consenso extendido sobre la necesidad de reformar los patrones de movilidad urbana en las ciudades contemporáneas. En este sentido, un documento recientemente publicado por la Unión Europea afirma que "el aumento del tráfico en las áreas centrales de pueblos y ciudades ha llevado a una situación de congestión crónica, con las múltiples consecuencias negativas que conlleva en términos de demoras y polución" (European Commission, 2007, p. 3). Muchos de estos problemas se encuentran relacionados con la predominancia en nuestras ciudades de un tipo particular de movilidad urbana: la automovilidad.

En términos de transporte urbano, la creciente relevancia de esta forma de movilidad se encuentra relacionada con el mantenimiento y/o desarrollo de una serie de problemas sociales mayores como (Bohm, Jones, Land, y Paterson, 2006, pp. 6-10):

- Congestión: en todo el mundo los automóviles son los principales partícipes de la congestión de tráfico.

- Sustentabilidad ecológica: el uso masivo de autos está relacionado con problemas ecológicos serios como el uso de recursos no-renovables, la polución del aire y ruido, entre otros.

- Problemas geopolíticos globales: el alto consumo global de petróleo ha desencadenado diversas crisis políticas, incluso llegando a desatar conflictos armados.

- Accidentes: los accidentes automovilísticos constituyen un problema social mayor, causando miles de muertes anuales así como también altos gastos públicos y privados.

- Exclusión social: "la falta de acceso a un auto es considerada una característica de la pobreza hoy, figurando en un buen número de índices de deprivación” (Kenyon, Rafferty y Lyons, 2002, p. 208).

Sin embargo pese a que las consecuencias negativas del uso creciente del automóvil se han conocido por un largo tiempo, hasta hoy la gran mayoría de las políticas públicas de transporte y medioambientales implementadas "han fracasado en cambiar el comportamiento y en llevar a cabo transformaciones sociales que conllevan cambios en tecnologías y hábitos" (Kemp y Rotmans, 2005, p. 33). En comparación con otras áreas de políticas públicas, "el sector de transportes ha probado ser un territorio particularmente duro para el avance de políticas de desarrollo sustentable" (Goldman y Gorham, 2006, p. 262). Como consecuencia, el número total y relativo de personas utilizando medios de transporte privados se ha incrementado en 
casi todas las ciudades del mundo en las últimas décadas (Steg y Gifford, 2005), especialmente en países en vías de desarrollo como China e India (Volti, 2008).

En el caso de la ciudad de Santiago (Chile) las tendencias no son diferentes. Múltiples estudios y publicaciones (De Mattos, Ducci, Rodríguez y Yáñez, 2004; Galetovic, 2006; Lanfranco, 2003; MINVU, 2007; SECTRA, 2002) nos muestran cómo la automovilidad, pese a no alcanzar aún los niveles encontrados en otras sociedades, ha experimentado una sostenida alza en las últimas décadas. Las causas de este fenómeno son diversas, como veremos a continuación, pero sus consecuencias son similares: aumento en la congestión, accidentes, polución ambiental, etc. Junto con esto, los problemas enfrentados en la implementación de Transantiago, el nuevo plan de transporte público de la ciudad, sólo han tendido a intensificar dicha tendencia. En este contexto existe una necesidad urgente de desarrollar políticas que efectivamente fomenten formas de movilidad urbana más sustentables, tanto en términos sociales como medioambientales. El presente artículo pretende contribuir a esta tarea con un estudio de las prácticas sociales y los significados detrás de la adquisición y uso de automóviles por parte de un grupo de habitantes de escasos recursos de la ciudad de Santiago, Chile.

\section{Automóviles, ciudad contemporánea y teoría social}

Lo primero que resalta al estudiar las relaciones entre automóviles y ciudad contemporánea desde una óptica de teoría social y urbana es, paradójicamente, el poco interés que ésta demostró por el tema hasta finales del siglo XX. Hasta ese momento el rol del automóvil fue minimizado, ocupando comúnmente un lugar meramente contextual de otros procesos y/o actores urbanos "realmente" centrales. Como consecuencia de esto,

“...los autos han sido concebidos o como una tecnología neutral, permitiendo patrones de vida social que hubieran ocurrido de cualquier forma, o como un monstruoso intruso que ha destruido patrones preexistentes de vida urbana. Los estudios urbanos no han considerado cómo los autos reconfiguran la vida urbana, implicando... formas distintas de habitar, viajar y socializar en y a través de un tiempo-espacio automovilizado" (Sheller y Urry, 2000, p. 738).

Como se desprende de la cita, este olvido contrasta con el hecho de que "pocas tecnologías desarrolladas en el siglo veinte han ejercitado un poder mayor sobre la imaginación popular, al mismo tiempo que amenazan la viabilidad de la existencia global, que el triunfo del automóvil sobre las sensibilidades culturales" (Carrabine y Longhurst, 2002, p. 181).

Con todo, desde el cambio de siglo han aparecido una serie de publicaciones y proyectos de investigación específicos que buscan subsanar en parte este olvido (Bohm et al., 2006; Cresswell, 2006; Featherstone, Thrift y Urry, 2005; Miller, 2001; Sheller y Urry, 2000; Urry, 2000, 2007). El punto de partida de muchos de estos acercamientos específicos al tema es el reconocimiento de que "la vida social en general ha estado irreversiblemente unida al modo de movilidad que la automovilidad genera y presupone" (Urry, 2004, p. 26). Esta particular forma de movilidad ha sido definida como "una amalgama compleja de máquinas interrelacionadas, prácticas sociales y formas de habitar, no un hogar estable, sino una cápsula móvil, 
semi-privada y altamente peligrosa” (Sheller y Urry, 2000, p. 739). Un elemento clave del concepto de automovilidad es la conciencia de que se debe abandonar "la idea del auto como una cosa, como un objeto singular de producción y consumo, y mirar a éste como un sistema de prácticas técnicas y sociales interrelacionadas que ha reconfigurado a la sociedad civil” (Gartman, 2004, p. 169).

Este énfasis en la automovilidad también nos debe llevar a cambiar la forma en la cual observamos y estudiamos las ciudades contemporáneas. Ello se debe principalmente al hecho de que "la automovilidad hace posible la separación del hogar con el lugar de trabajo, de los distritos de negocios e industria con los hogares, de los lugares de venta con los centros de las ciudades" (Featherstone et al., 2005, p. 2). Sin embargo para que estas múltiples divisiones y especializaciones del espacio urbano puedan llevarse a cabo, la ciudad misma debe transformarse radicalmente. La relevancia de esta transformación es tal que se ha llegado a afirmar que el automóvil "ha sido el factor más significativo en la transformación de la forma urbana contemporánea” (Taylor, 2003, p. 1612).

Como señalan Graham y Marvin (2001), para poder soportar este número creciente de automóviles (junto con una serie de otras movilidades: comunicaciones, energía, desechos, etc.) las ciudades deben transformarse en un proceso, en un "punto de abastecimiento de oleadas perpetuas de flujos, movimientos e intercambios mediados por infraestructuras" (p. 14). Taylor (2003, p. 1613) identifica cinco tipos de transformaciones que el desarrollo de la automovilidad ha generado en los espacios urbanos en los últimos cincuenta años:

- El impacto visual de los automóviles en sí mismos, como objetos materiales, que transforman la tradicional geometría y disposición espacial de las calles.

- La transformación de áreas crecientes de la ciudad y su entorno por la construcción de infraestructura vial, desde nuevas calles a túneles y carreteras urbanas.

- El aumento en el tráfico ha llevado a una sobrepoblación de signos y equipamiento urbano (como los semáforos) para controlarlo.

- Para acomodar a los autos detenidos, significativas porciones de calles y edificios han sido dedicadas a proveer estacionamientos.

- Finalmente todos estos elementos han cambiado la estructura misma de la ciudad, permitiendo su extensión territorial y la fragmentación del espacio urbano.

Esta nueva visión de ciudad como proceso, como un flujo constante de movilidades múltiples, debe observarse como si operara de forma traslapada al tradicional concepto de ciudad como una entidad estable.

"Si la urbanización lleva a la intensificación de los hábitat humanos, la concentración de lugares en el espacio y la unificación de flujos temporales condensados, la automovilización, en contraste, lleva a la extensión de los hábitat humanos, la dispersión de lugares en el espacio, la oportunidad de escapar de ciertas localidades y formar nuevas socialidades y la fragmentación de los flujos temporales, especialmente a través de la suburbanización" (Sheller y Urry, 2000, p. 742). 
Urbanización y automovilización entonces pueden ser vistas como formas diferentes de habitar los espacios urbanos contemporáneos. Éstas no se oponen, sino que, por el contrario, se necesitan mutuamente para poder existir. De hecho, se puede afirmar que históricamente ambas han sido co-construidas, es decir, aparecen como resultado de los mismos procesos sociales. Por un lado, la automovilidad aparece, especialmente desde mediados del siglo XX, como un correlato necesario de ciudades en las cuales los números crecientes de población y el aumento territorial van de la mano con la necesidad de movilizar a un número mayor de personas y bienes de la manera más rápida y personalizada posible. Por su parte, la progresiva disponibilidad de tecnologías de transporte personal, especialmente en forma de autos, se relaciona crecientemente con el desarrollo de formas de urbanidad cada vez más segmentadas, fluidas y en constante expansión territorial.

Junto con lo anterior es importante mencionar que la relevancia urbana del automóvil va más allá de sus conexiones con el ámbito de lo territorial/material. A un nivel individual hay que tomar en cuenta que "al reconsiderar el concepto del automóvil es importante reconocer que su rol no es puramente funcional en el sentido de mover pasajeros de un lugar a otro en una forma conveniente y autónoma" (Wright y Curtis, 2005, p. 14). Junto con su utilización práctica para movilizarse por las ciudades contemporáneas, el automóvil también representa uno de los íconos culturales más significativos de la sociedad actual. Desde su lugar como objeto de campañas publicitarias millonarias hasta el hecho de ser un tópico recurrente de conversación, el automóvil ocupa un lugar central en las prácticas sociales a través de las cuales se construyen una serie de categorías clave como el status social, la estética, el poder, etc. Como resultado de esta situación, incluso "para aquellos que no tienen uno o no tienen acceso continuo a uno, 'el auto' como un objeto da forma continua a nuestras vidas a través de su efecto penetrante y multifacético en nuestra cultura” (Dant, 2001, p. 61).

\section{Características del estudio y métodos}

El trabajo en terreno sobre el cual se basa el presente artículo fue llevado a cabo durante catorce meses entre 2007 y 2008 en la ciudad de Santiago, Chile. Más específicamente, el estudio se enfocó en un grupo de familias de ingresos medios-bajos y bajos que habitaban en cuatro sectores de la ciudad. Estas locaciones se seleccionaron en función de dos criterios clave: la presencia en ellas de un número importante de familias de clase media-baja y baja (que al menos un tercio de su población se ubicara en el primer y segundo quintil de ingreso), y su ubicación geográfica central o periférica respecto del centro de la ciudad. Como resultado de la aplicación de estos criterios se seleccionaron los siguientes sectores:

- Villa El Salto (comuna de Recoleta)

- Conjunto Carlos Walker Martínez (comuna de San Miguel)

- Villa Isabel Riquelme (comuna de Maipú)

- Villa Chiloé (comuna de Puente Alto) 


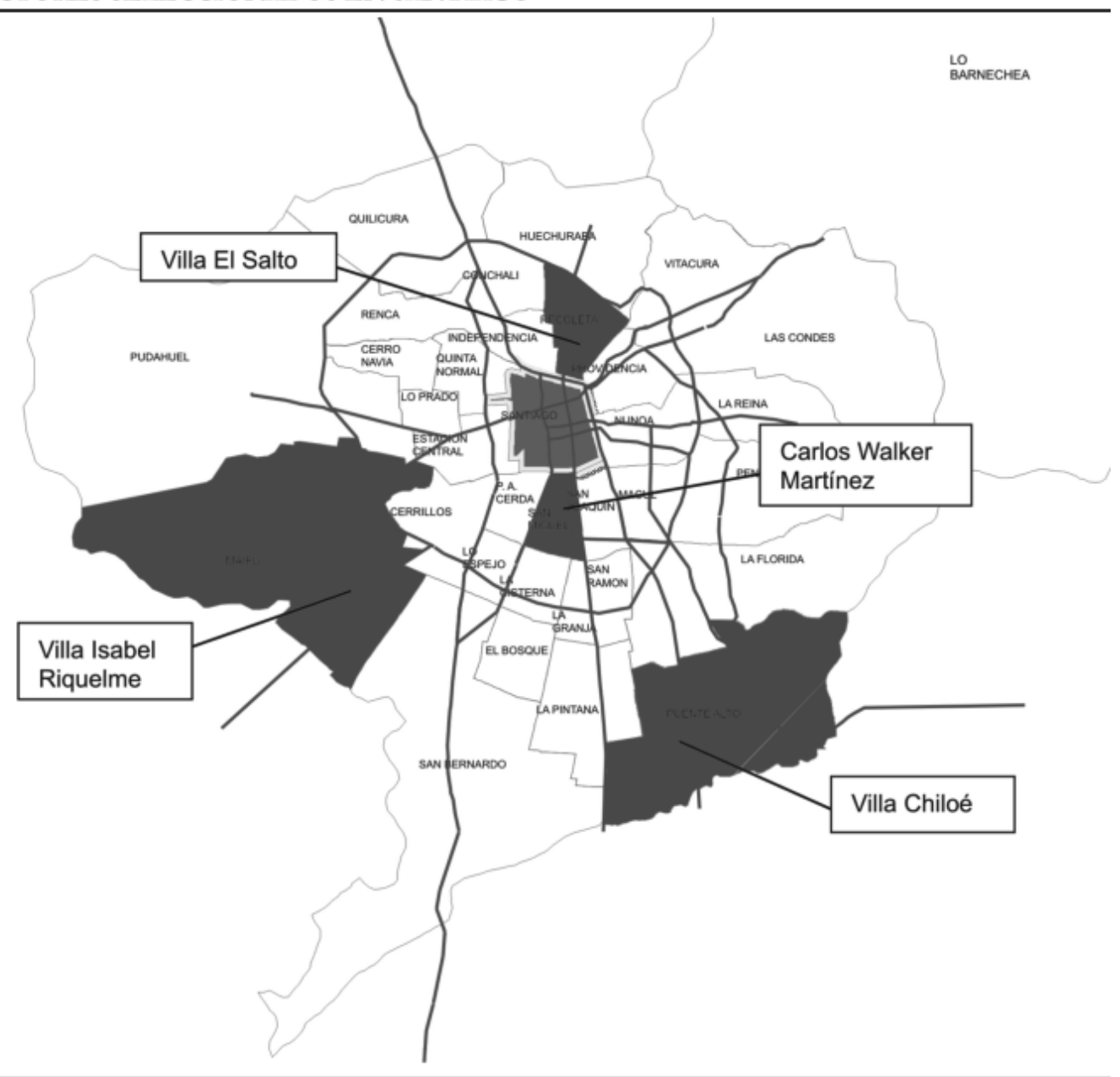

Fuente: Elaboración propia

En cada uno de estos sectores se seleccionó a un grupo de cinco familias. En términos de sus características socioeconómicas la mayoría de éstas puede ser descrita como teniendo un ingreso relativamente bajo, usualmente con sólo uno de los padres trabajando en un empleo de baja calificación dados sus pocos años de educación formal. En términos de género, edad, actividad y número de miembros se optó por seleccionar la mayor variedad posible, desde parejas de adultos mayores viviendo solos hasta familias de más de diez miembros viviendo en situación de allegamiento. El último factor tomado en cuenta al seleccionar los casos fue que en cada sector hubiera familias con usos diferenciados de tecnologías del transporte. En todas ellas había miembros que usaban de manera regular el transporte público y en nueve casos también tenían automóvil o furgón en su hogar.

En términos de metodología, esta investigación se basa en un acercamiento de tipo cualitativo basado primariamente en series de entrevistas en profundidad, realizadas en el hogar de cada familia y estudiando a todos sus miembros mayores de diez años. Junto a lo anterior se realizó 
observación participante tanto en el hogar de las familias como en sus viajes cotidianos, la cual fue registrada con fotografías y notas de campo. Por último es relevante hacer notar que todos los nombres han sido cambiados para proteger el anonimato de los participantes en el estudio.

\section{Movilidad espacial y automóviles en Santiago}

Hoy en día constituye casi un lugar común afirmar que la ciudad de Santiago ha cambiado radicalmente en las últimas décadas. Extensión territorial y crecimiento poblacional, nuevas carreteras y líneas de metro, masivo acceso a vivienda social, nuevos barrios, centros de negocios y comercio son algunos ejemplos de cambios que han transformado el entramado urbano de la ciudad (De Mattos et al., 2004; De Mattos e Hidalgo, 2007; Galetovic, 2006; INE, 2003; Ramos, Valenzuela, Larrañaga, Medrano y Gubbins, 2004; SECTRA, 2002). Al centro de muchas de estas transformaciones podemos encontrar un elemento unificador: la creciente importancia de la movilidad espacial. Desde el acceso a bienes de consumo masivo hasta la forma en la cual nos contactamos con nuestros seres queridos, es difícil encontrar procesos urbanos en los cuales algún tipo de movilidad de objetos o personas no cumpla un rol determinante.

La relevancia de esta movilidad se explica por los cambios que ha sufrido la ciudad en la última década. Como señala Ducci (2002), el área urbana de Santiago en 2002 cubría 61.395 hectáreas, lo cual representa un aumento de 1.339 hectáreas por año durante la década de los noventa, el crecimiento más alto de su historia. Un punto central es que el $40 \%$ de este crecimiento se explica por la construcción de casas, casi el doble del terreno usado por la industria (23\%). Es decir, cada vez más gente habita las nuevas áreas de la ciudad, quedando alejada de los centros urbanos tradicionales y viéndose obligada a desplazarse distancias cada vez mayores para llegar a ellos. Esto es especialmente cierto para el importante grupo de la población de menores ingresos que vive en vivienda social, ya que dichos conjuntos suelen ser construidos en los límites del área urbana de la ciudad (Hidalgo, 2007; Tironi, 2003). Esta ubicación, alejada de los centros de trabajo, estudio, esparcimiento y sociabilidad, transforma a la movilidad espacial en uno de los factores claves de participación en la dinámica urbana actual de la ciudad.

Tal transformación en el entramado espacial de la ciudad y su efecto en términos de la fragmentación y extensión territorial de las actividades diarias, no hubieran sido posibles sin la existencia de cambios correlativos en el otro ámbito clave de la dinámica urbana identificado en la sección anterior: la automovilidad. Estos cambios se ven reflejados tanto en el aumento sostenido de la posesión y el uso de automóviles para viajes diarios como en la construcción de una serie de obras de infraestructuras de movilidad espacial. Respecto al primer punto, los datos disponibles nos muestran que mientras en los quince ańos que pasaron entre 1977 y 1991 la relación de vehículos por hogar sólo creció marginalmente (de 0.32 a 0.36), en la década siguiente ésta explotó hasta alcanzar los 0.56, lo que significa que en el año 2001 más de la mitad de los hogares de la ciudad de Santiago tenía alguna forma de vehículo motorizado (SECTRA 2002). Esta tendencia se ve corroborada por los datos entregados por el INE que muestran un alza del 5,5\% en el parque automotor de la Región Metropolitana solamente 
entre los años 2006 y 2007, concentrando actualmente el 43\% (1.200.000 aprox.) de los automóviles en circulación en el país (INE, 2008). Respecto del segundo punto, desde mediados de los años 90 el Ministerio de Obras Públicas ha estado involucrado en un ambicioso plan de construcción de infraestructura de movilidad urbana a través del modelo de autopistas urbanas concesionadas a privados, de las cuales los seis principales proyectos sumaban 215 kilómetros de extensión en el año 2005 (Greene y Mora, 2005). Estas autopistas junto con otras obras viales concesionadas han modificado de manera importante la forma en que se materializan los regímenes de automovilidad en la ciudad.

Un punto relevante es que más allá de la desigualdad que caracteriza a la sociedad chilena actual, y que se manifiesta en un acceso altamente diferenciado a tecnologías por grupos socioeconómicos, los segmentos de menores ingresos no han sido ajenos a estos procesos de cambio. Por ejemplo, los datos disponibles muestran que, pese a que sólo el $10 \%$ de los hogares pertenecientes a estos grupos tiene a su disposición alguna forma de vehículo motorizado, tal posesión presenta en el periodo 1992-2002 una tasa de aumento que es casi el doble de la que muestra la población general (Larrañaga, 2004). Esta idea se refuerza al analizar los datos entregados por la encuesta CASEN que se presentan en la siguiente figura. Como se puede observar, si bien en el periodo entre las encuestas de 1990 y 1998 todos los quintiles de ingreso aumentan de manera muy importante su posesión de automóviles, en el periodo 1998-2006 esta tendencia desaparece (e incluso parece revertirse) en los hogares de sectores medios y altos, mientras que para los hogares del primer y segundo quintil esta continúa siendo positiva.

Figura 2

POSESIÓN DE AUTOMÓVILES POR QUINTILES DE INGRESO EN HOGARES

DE LA REGIÓN METROPOLITANA DE SANTIAGO, CHILE

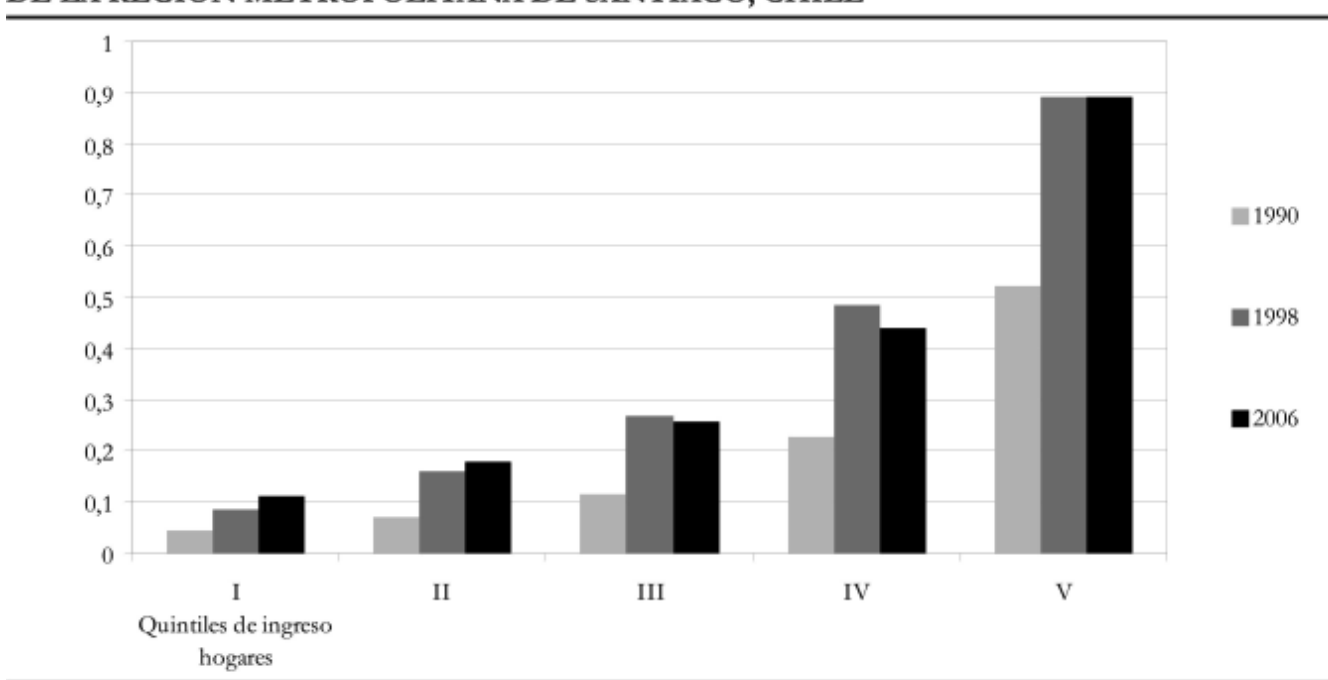

Fuente: Elaboración propia basada en resultados de encuestas Casen 1990, 1998 y 2006

Estos datos, pese a ser sólo parciales, nos pueden llevar a hipotetizar que será precisamente este segmento el que aumentará mayormente su participación en formas de automovilidad durante los próximos años, con todas las consecuencias que se detallaron en la introducción. A continuación pasaremos a describir y analizar en profundidad los principales hallazgos 
respecto a las razones de este proceso de adquisición y su efecto sobre las prácticas cotidianas de movilidad espacial en el grupo de familias bajo estudio.

\section{Sentido y utilización del automóvil en la vida cotidiana}

\section{Adquisición}

Como ocurre en relación a diversos otros bienes de consumo masivo, para los miembros de las familias estudiadas la llegada del primer automóvil se asocia comúnmente con una serie de emociones intensas y a la satisfacción de haber logrado un proyecto largamente deseado. Tomás, un hombre de 58 años que vive en la villa Isabel Riquelme de Maipú, nos relataba de esta manera la llegada de su camioneta:

-...siempre soñé yo con tener un vehículo y a la edad que lo viene uno a tener, a los cincuenta y siete.

- ¿Y por qué era un sueño?

Porque nunca tuve la oportunidad de tener un vehículo y siendo que yo fui toda la vida chofer, fui mecánico primero y después fui chofer... y ya cuando tuve la oportunidad, se me dio y hicimos el sueño con la vieja, lo hicimos realidad... esa camioneta me la compró mi hijo, él me hizo unos movimientos en su trabajo y la compró él en su trabajo.

- ¿Y cómo fue cuando llegó la camioneta?

No, si nos llevó a buscarla... Ahhh... casi nos caímos de espalda cuando la vimos... emocionante, un vehículo grande, bonito, cómodo, rápido.

En este extracto podemos ver cómo, especialmente en segmentos que tradicionalmente estuvieron excluidos del acceso a este tipo de tecnologías, la posesión del automóvil y su utilización "ofrece a muchas personas un sentimiento de liberación, empoderamiento e inclusión social" (Sheller, 2004).

Sin embargo, la importante carga de satisfacción que usualmente conlleva la adquisición de un automóvil no implica necesariamente que ésta sea resultado de un proceso de planificación racional por parte de sus usuarios. Por el contrario, uno de los elementos que aparecen como más destacados de este proceso es su carácter contingente en el sentido de que la llegada del auto al hogar en muchos casos responde a circunstancias u oportunidades que aparecen de manera casi fortuita en un determinado momento.

Esto es bastante claro cuando vemos el caso de Mariana, una dueña de casa de 47 años que habita en la villa Chiloé, respecto de su auto, un viejo Datsun de 1976:

— ¿Cómo es la historia del auto? ¿Cómo llegó a ustedes?

Mi suegro... nosotros teníamos un auto antes y un día mi suegro hizo que mi esposo lo fuera a dejar a Padre Hurtado, porque nosotros tenemos familia allá y él no quería ir, no quería ir y fue, se pusieron a tomar por aquí, por allá y lo pararon en Talagante y llevaron a mi cuñado 
y a él y le quitaron el auto, entonces perdimos ese auto y nunca pudimos retirarlo... estuvo una semana y salía más caro los corrales que el auto... eran cinco lucas diarias en ese tiempo... por eso lo perdimos el otro auto nuestro porque nos había costado tanto, y entonces después mi suegro se enfermó, lo trajimos a vivir acá y cuando él murió aquí en mi casa dejó el auto, aparte que este auto es historia aparte...por eso lo tuvimos porque si no lo hubiéramos tenido no lo hubiéramos comprado más tampoco, porque ya los años pasan y uno ya no tiene la misma fuerza que antes que trabajaba.

En el caso de Mariana aparece como una especie de retribución de su suegro por los cuidados que le prestaron al enfermarse y su participación en la pérdida del automóvil anterior. El carácter fortuito que la adquisición del automóvil tuvo en los casos estudiados nos muestra cómo muchas veces los procesos a través de los cuales las personas adquieren tecnologías y las incorporan a sus vidas no responde a una cierta necesidad preestablecida, racionalidad económica o la búsqueda de "distinción social", sino simplemente a un elemento de contingencia que hace accesibles los bienes en un determinado momento del tiempo.

Como veíamos en la sección anterior, el parque automotor en la ciudad ha aumentado de manera considerable, presentando tasas de crecimiento mayores al 5\% anual en los últimos ańos. Esta tendencia tiene por consecuencia que se produzca un cierto flujo de autos de segunda mano, desde usuarios de mayores ingresos a estos segmentos. Este hecho, sumado a la mejoría general en las condiciones de vida de los grupos de menores ingresos, hace que aumenten de manera importante las oportunidades contingentes de adquisición de automóviles por parte de estas personas.

Sin embargo, por mucho que la posibilidad de adquirir un automóvil se presente de manera más frecuente y casual, ésta no tendría un real impacto en los niveles de automovilidad del segmento estudiado si no fuera porque, obviamente, tales bienes pueden ser adquiridos. Debido a su alto precio, aun en términos relativos, estas familias comúnmente no pueden acceder a pagar en efectivo por automóviles, incluso por aquellos de muy bajo precio (como se vio en el caso de Mariana). En este contexto la posibilidad de adquirir un automóvil aparece mediada por la disposición de uno de los mecanismos centrales que explican el cambio en los patrones de consumo de la población chilena de ingresos bajos: el crédito.

Esto queda bastante claro en el caso del nuevo auto de la familia Pérez, habitantes de la Villa Chiloé, como nos señalaba Mariela (29 años) en una de las entrevistas.

- ¿Y el auto? ¿Cómo lo compraron?

Mira, él vendió el que tenía y la gran diferencia... nos conseguimos un préstamo, yo me conseguí un préstamo en el banco París y ahí compramos el auto en una parte súper buena, se lo vendió un jefe del trabajo... ¿Cachai? entonces le dio buen precio y le tuvo que pagar, le faltaron ciento cincuenta mil pesos y eso se lo pagó en cuotas, que fueron tres cuotas de cincuenta mil pesos, pero el resto se lo dio todo así, lo que tenía guardado más lo que vendió el auto, nos costó harto comprar el auto... Pero ya es de nosotros ¿cachai?, ya está pagado, ya éste no lo vende. 
Como ocurre en otros contextos, la posibilidad de optar a créditos aparece como una de las principales herramientas a través de las cuales estas personas adquieren bienes de mayor costo como automóviles. Sin embargo, esta forma de acceder al objeto también tiene su contrapartida en el riesgo del endeudamiento (que afecta a un número importante de estas familias) y en el hecho de que el gasto en el automóvil tiene que insertarse en un contexto mayor, el de los gastos necesarios para mantener a la familia. Como nos señalaba Joanna (26 ańos), quien es madre de dos hijos y vive de allegada con sus padres en Recoleta:

- ¿Y acá en la casa no han pensado en comprarse un auto?, ¿no han conversado el tema? [Mi papá] ahora dice que no porque las cosas no están buenas como para invertir en un auto... Es que los autos son caros, igual se puede sacar a cuotas pero es que nosotros gastamos mucho, imagínate darle de comer a mi hija, pañales, él va al colegio, los colegios son carísimos ¿cachai? .... yo cacho que todas las personas sueñan con tener auto, todos cuando tienen 18 años dicen... yo con mi primer sueldo voy a ir y me voy a comprar un auto, aunque no me alcance ¿cachai?, ésa es la típica del chileno, así como las mujeres... yo me quiero casar de blanco, pero todo chileno quiere tener un auto o toda persona en el mundo dice yo quiero mi auto' ¿cachai?, pero tengo que privilegiar mi comida...

Este extracto grafica bastante bien las tensiones detrás de la adquisición de automóviles por parte de los miembros de estas familias. Por un lado el auto aparece como un bien de consumo ampliamente deseado ("todas las personas sueńan con tener un auto"). Por otro lado encontramos la realidad de un presupuesto reducido en el cual el gasto que implica tener un automóvil (sin siquiera considerar los gastos de mantención y uso de éste) aparecen como limitando la posibilidad de satisfacer necesidades básicas como alimentación y educación.

\section{Prácticas de uso cotidianas}

La primera práctica relativa al uso de automóviles que aparece mencionada por los individuos bajo estudio son los viajes al trabajo del jefe de hogar, como reconoce Paula.

- ¿Y la camioneta para qué la ocupan?

Más para el trabajo de mi marido... la camioneta está como destinada para la fuente de trabajo... mi marido trabaja independiente, él es vendedor ambulante.

- ¿Y es importante para su marido la camioneta?

Sí, importante, por ser él tiene que andar con un carrito, una cajita, entonces la micro no lo lleva y ahí se moviliza (Paula, 42 años, Puente Alto).

Como se mencionó anteriormente, en la gran mayoría de los hogares bajo estudio solamente uno de sus miembros realiza alguna tarea remunerada, comúnmente hombres. En un número importante de casos estos trabajos son bastante informales y precarios (como el trabajo de vendedor ambulante de Patricio, el marido de Paula) o, debido a las bajas remuneraciones, tienen que ser complementados con trabajos secundarios informales, los "pololos". En ambos casos la capacidad de movilizarse de una manera flexible característica de la automovilidad es clave para lograr acceder a las oportunidades disponibles. 
Un segundo uso que se menciona de manera frecuente son los viajes a realizar trámites y compras, especialmente durante los fines de semana, como mencionaba Jonathan (47 años, Recoleta).

- ¿Con qué frecuencia anda usted en auto? ¿En qué situaciones?

Todos los domingos, de repente el sábado, cuando voy al mall, voy de compras, voy al centro, voy al cine de repente, voy en auto... de mi sobrino, de mi amigo, de mi compadre, o sea tengo la suerte que siempre hay un auto en la casa, el de mi hermano, de mi sobrino, compadre.

- ¿Y lo usa básicamente, para qué?

Para ir de compras, nada más.

Este tipo de usos de los automóviles, especialmente para acceder a centros comerciales de construcción reciente, en muchos casos no responde solamente a la necesidad de comodidad o velocidad. Por el contrario, muchas veces los centros comerciales y la infraestructura urbana que los rodea presupone de manera casi compulsiva el uso de algún vehículo motorizado para llegar a ellos (Graham y Marvin, 2001).

Finalmente un último uso relevante que se le da al automóvil es en relación al tiempo libre, tanto en términos de visitas a familiares y amigos, como a viajes de tipo recreacional, dentro y fuera de Santiago, como reconoce Alan, un hombre casado de 59 años que vive con su familia en la villa Chiloé.

- ¿Y qué significa el auto para usted?

Eso es súper importante, es un gran avance que se tiene, es una gran independencia porque uno ya no está dependiendo si... iré a encontrar micro o tengo que irme porque voy encontrar la última micro, ya no pasa eso, uno depende de sí, de sí mismo, uno decide hasta qué horas se queda, a qué hora se va, si va a lugares lejos donde no hay locomoción, cuando sale la noche, tengo familiares en Alto Jahuel., de Buin hacia el interior, hacia la cordillera, o vamos donde mi hija también allá a [la villa] Portales y uno se viene a la hora que quiere, entonces esas son las facilidades de tener auto... Viajamos mucho a visitar a los familiares de mi señora y mucho a Molina y a Talca. Pero el año pasado nos pegamos un viaje con mi señora y mi hermano y la señora de mi hermano a Chiloé.

En el caso de Alan, la posesión de un automóvil le ha permitido realizar una serie de actividades recreacionales, tanto en términos de visitas a familiares como puramente turísticas. Como él reconoce, probablemente varias de estas actividades se podrían haber realizado de la misma manera si ellos no tuvieran acceso a un vehículo, pero éste les permite una independencia y flexibilidad que no podrían encontrar en un medio de transporte de tipo colectivo, especialmente en el caso de viajes largos como las vacaciones a Chiloé en el sur de Chile. En ese sentido, la automovilidad toma un rol diferente en el proceso general de mejora en las condiciones de vida que las personas de menores ingresos de Chile han experimentado en los últimos ańos, apareciendo no solamente como el acceso a una forma de movilidad de la cual estos grupos estuvieron tradicionalmente excluidos, sino también como un capacitador de la 
realización de actividades, como el turismo y el ocio, que tienen un peso cada vez mayor en la autoevaluación de la calidad de vida personal de los miembros de estos segmentos.

Sin embargo, estas nuevas formas de automovilidad de tipo recreativo son bastante sensibles a cualquier fluctuación en sus condiciones, especialmente en términos monetarios. Esto queda claro en el siguiente extracto de la entrevista con Carla:

Antes lo ocupábamos harto, viajábamos a Olmué, salíamos bastante; ahora no, con el alza de la bencina y esta cuestión se sale poco, solamente por emergencia, o sea ir a comprar, o sea que esté enfermo uno de los papás eh... por decirte, para solamente emergencias, ir a comprar, pero no así como para darnos viajes (Carla, 40 años, Recoleta).

Ante el aumento en el precio de uno de los insumos básicos de la automovilidad como es la bencina, situación mencionada por varios de los individuos bajo estudio, los patrones de viajes se contraen de manera sensible a lo que se percibe como completamente necesario (emergencias y compras en el caso de Carla), casi eliminando cualquier forma de movilidad de tipo recreativo.

Finalmente es relevante destacar que en el uso cotidiano del automóvil no todos los miembros del hogar tienen los mismos roles. Por el contrario, existe una clara demarcación interna entre diferentes tipos de usuarios. Esta demarcación está dada por el rol completamente pasivo que tienen las mujeres en las prácticas de automovilidad de estas familias. En la totalidad de los casos bajo estudio el único rol que las mujeres de la casa tenían era el de pasajeras en el auto, casi siempre en días no laborales; ninguna de ellas sabía realmente manejar, ni menos aún tenía licencia de conducir. Exploraremos este punto con el caso de Juana.

Ella tiene 35 años y vive en un departamento del conjunto Carlos Walker Martínez (San Miguel). Al momento de realizar este estudio se encontraba soltera y vivía con sus dos hijos. A diferencia de muchas otras mujeres había tenido la oportunidad de hacer un curso de manejo, pero los resultados habían sido bastante poco satisfactorios.

- ¿Te gustaría tener un auto?

Sabes que hice un curso de manejo, yo hice un curso de manejo pero no sirvo para manejar porque soy muy histérica, nerviosa, pero yo creo que me puedo acostumbrar, lo único que quieren es que tenga auto [hijos], porque, claro, ellos están acostumbrados, los papás tienen autos...

- ¿Entonces a ti no te gustaría tener un auto?

No porque no soy muy nerviosa, entonces cuando hice el curso de manejo, la práctica bien, pero de repente el instructor nos hacía venir aquí a Panamericana, entonces de repente venía un camión y ¡Aaaaaaa!... Después que hice el curso, no sé qué paso es que se me paró muchas veces el auto, pasé todas las pruebas, pero de práctica se me paró como tres veces el auto, me dijeron venga a repetirlo porque igual está bien, pero no... nunca fui. 
Esta relativa exclusión femenina del mundo de la automovilidad no es algo nuevo. Desde su invención hasta mediados del siglo XX los automóviles fueron definidos como una tecnología eminentemente masculina, "tanto porque estos proveyeron movilidad en ámbitos públicos como porque eran objetos de producción mecánica y utilitaria” (Gartman, 2004, p. 174). Sin embargo esta situación cambió radicalmente desde mediados de siglo, cuando una serie de transformaciones en las actividades y el status de las mujeres (integración al mundo del trabajo, movimientos feministas, etc.) las llevaron a ver que "sus miedos iniciales del nuevo medio de transporte era mínimos en comparación con las potenciales ventajas que adquirían con él y urgieron la adquisición de un vehículo familiar cuando era financieramente viable" (Walsch, 2008, p. 382). Como resultado de estos procesos actualmente "para muchas mujeres el automóvil es central para la logística de mantener las relaciones mundanas del hogar" (Featherstone et al., 2005, p. 14).

En el caso bajo estudio podemos observar una situación similar. Debido a la relativa novedad del automóvil en el contexto cotidiano de estas familias y a la persistencia de distinciones de género tradicionales en términos de competencias y roles, esta tecnología es todavía percibida como un artefacto masculino. Lo relevante es que esta percepción es sostenida mayoritariamente por la mujeres mismas, que frecuentemente tienden a descalificarse cuando dan las razones de por qué no manejan ("soy muy nerviosa"). Sin embargo podemos hipotetizar que esta situación va a tender a cambiar rápidamente a medida que la tecnología se vuelva una parte normal de la vida cotidiana de estos grupos. Este cambio probablemente se va a reflejar en un aumento sustantivo de los grados de automovilidad actuales de estos segmentos, lo cual impactará de manera importante en el régimen general de movilidad urbana existente en Santiago en la actualidad.

\section{Valoración y sentido del automóvil}

Como podría esperarse, mantener el auto funcionando y su uso cotidiano representa para estas familias un proporción bastante alta de sus ingresos mensuales. Por ejemplo Mariela nos hablaba del automóvil de su hogar como una "segunda casa":

— ¿Cómo lo hacen para mantenerlo, para pagar los costos?

Es que mi marido gracias a Dios tiene buen sueldo... O sea que nos abastecemos bien nosotros como familia, como casa, y más encima la segunda casa que es el auto, porque te gasta, o sea tenís que tenerlo en buenas condiciones para que el auto...

- ¿Entonces es como la segunda casa, el auto?

Yo siempre he opinado eso... Porque, qué se yo, que cada cierto tiempo tiene que cambiarle los neumáticos, cada cierto tiempo tení que hacerle cambio a los niños, que pucha se te quemó una lucecita ¿cachai?, o no sé $p o$ ', ayer se le soltó una abrazadera de la bomba de agua y estaba botando agua ¿cachai? (Mariela, 29 años, Puente Alto).

Para Paloma, en la misma línea, el automóvil de su hogar era una especie de "amante": 
“...tener un vehículo es como tener una amante le digo yo a mi marido, si yo pido no se me cumple rápidamente, pero si al auto le falta, no sé, una bujía, un neumático, hay que cambiarlo altiro porque es el medio que nos transporta, pero si yo necesito una tintura pa'l pelo, 'espera el próximo mes', pero el auto no espera y por eso le digo que es como tener una amante, le digo yo". (Paloma, 50 años, Puente Alto)

Ambas imágenes, el auto como "segunda casa" y "amante", nos hablan de una tecnología que conjuga, por un lado, una importante carga emocional para sus usuarios, y por el otro, implica una serie de gastos que exceden de manera importante los de otros bienes equivalentes. Como mencionan Sheller y Urry, "el consumo del auto no es nunca simplemente una opción de racionalidad económica, sino que es también sobre respuestas estéticas, emocionales y sensoriales al acto de manejar, así como también patrones de parentesco y sociabilidad, de habitar y trabajar" (2000, p. 222). Este rasgo del automóvil y la automovilidad como práctica fue bastante evidente cuando se exploraban en las entrevistas las razones detrás de la posesión de vehículos.

La respuesta más usual estaba relacionada con el ahorro de tiempo que el automóvil supuestamente representaba respecto a otras formas de movilidad urbana, especialmente el transporte público, como reconoce Rodrigo.

— ¿Te gustaría tener un auto acá en tu casa?

Sí... por comodidad, porque por el sentido que uno ahorra tiempo, ahorra tiempo y tienes la facilidad de poder elegir dónde ir un fin de semana, sin preocuparte de que hay que levantarse muy temprano, hay que andar corriendo en las micros y con todo lo que conlleva ese tipo de viaje... que hay que andar a horario y en cambio en auto no $p o^{\prime}$, uno si quiere se levanta un poquito más temprano por ejemplo si quiero ir a Vińa, no sé $p o$ ', por ponerte un caso ya los vamos a Viña a las seis de la mañana que los levantemos no es lo mismo que levantarse a las cinco para ir a tomar el bus allá a Estación Central y lo entretenido que cuando vamos a la playa el bus pasa por acá y hay que pegarse el pique de una hora para allá y después media hora para acá de vuelta para pasar por acá mismo porque, porque acá no es paradero (Rodrigo, 38 años, Maipú).

Este tipo de argumentación se conecta de manera central con la progresiva adopción por parte de los segmentos de menores ingresos del país de maneras de valorar el tiempo características de la sociedad moderna (Nowotny, 1994). Como se vio en un estudio anterior (Ureta, 2006), los grupos de menores ingresos tradicionalmente tendían a percibir el tiempo como un recurso medianamente abundante (o al menos más abundante que otros segmentos de la población). Sin embargo los cambios que las condiciones de vida de estos individuos han experimentado en los últimos años (integración creciente al mundo laboral y la educación, mayor formalización de actividades, etc.) han hecho que tiendan a adoptar patrones de valoración temporal cada vez más cercanos a la norma moderna del tiempo como un "recurso escaso".

En este contexto el automóvil es percibido (así como también construido en la publicidad y el marketing) como la tecnología que nos va a permitir aprovechar este tiempo escaso. De hecho, desde su origen a principios de siglo "una de las promesas centrales del automóvil 
fue que nos iba a liberar de los límites espacio-temporales" (Hagman, 2006). Entonces la principal motivación que encontramos detrás de la automovilidad, claramente presente en la evaluación de Rodrigo, es esta idea moderna de que la "automovilidad por tanto genera un tiempo 'instantáneo' o 'a-temporal' que puede ser mezclado y manejado de formas complejas, heterogéneas e inciertas” por cada individuo (Sheller y Urry, 2000, p. 744).

Esta idea del ahorro temporal que supuestamente el automóvil permitiría (y que en muchos casos no pasa de ser un mito) se interrelaciona con otra línea de argumentación de uso frecuente: la comodidad. Esto queda claro en el siguiente extracto de una de las entrevistas con Alan:

— ¿Por qué compró su primer vehículo?

Por la comodidad que te permite, si la razón siempre es ésta, es la comodidad que tú sabes que te va dar tener un vehículo, toda la independencia que te da, dejas de ser dependiente de la locomoción colectiva y de muchas cosas y además que te da mucho a... evita mucho trabajo, mucho esfuerzo, el ir al supermercado, el ir de compras y no tan sólo supermercado sino que muchas partes que uno necesita tener que comprar algo... entonces esas son las facilidades de tener auto, ya no tenís que ir al mercado y andar cargado de bolsas, o sea hay muchas cosas que te estoy dando algunos ejemplos, el salir de viaje, andar de vacaciones y no andar cargado metiéndote al bus lleno de maletas, pescas todas las cuestiones y te vas" (Alan, 59 años, Puente Alto).

Esta argumentación sobre el confort tampoco es casual. Como ha señalado Crowley (1999), desde el siglo XVIII la idea del confort se ha erigido como un valor típicamente de clase media, proveyendo éste "la racionalidad para patrones de consumo moderado pero innovadores que trascendieron los imperativos aristocráticos por el lujo y las necesidades de la pobreza" (p. 21). Desde esta perspectiva, la idea de "viajar confortable" en automóvil versus las limitaciones asociadas al uso del transporte público, también puede interpretarse como un indicador del nuevo estatus social de los grupos sociales bajo estudio debido a las mejoras en las condiciones de vida en las últimas décadas.

Una tercera línea de argumentación respecto a la necesidad de la automovilidad tiene que ver con la mayor accesibilidad que el automóvil otorga a diversos lugares. En los casos bajo estudio este argumento por la accesibilidad se encarna preferentemente en hipotéticas situaciones de riesgo que los miembros del hogar u otros vecinos pueden sufrir y que requerirían el acceso rápido a centros asistenciales, como nos relataba Mariela:

- ¿El auto es importante para ustedes?

El auto, sí... es importante porque mira, nosotros aquí estamos tan lejos de todo, claro que si tú sales en viaje... paseo de placer, es súper rico salir en una micro o salir en metro, qué sé yo, es re entretenido salir, caminái', que sé yo... pero si tú tení que salir de emergencia eso te queda, pero se te hace eterno el viaje po` o sea yo tuve a cargo una guagüita... Me la estaban viendo en el hospital, la estaba viendo justo la doctora, tuve una sobrina a cargo nueve días... ella se enfermó, yo tuve que ir así al médico, yo pensé que no iba a llegar nunca ¿cachai?, entonces claro, el auto no está en el día acá... pero sirve cuando se te enferman los niños, 
lamentablemente casi siempre es de noche, en la tarde cuando se enferman más, entonces cualquier cosa nosotros partimos (Mariela, 29 años, Puente Alto).

Esta línea de argumentación puede ser analizada desde dos perspectivas complementarias. En primer lugar, la accesibilidad ha sido conectada con situaciones de exclusión social en diversas ciudades a nivel mundial (Cass et al., 2005) y Santiago no es una excepción a esta tendencia (Jirón, 2007; Ureta, 2008). Una de las características centrales de muchos barrios y zonas de ingresos bajos es su difícil accesibilidad al resto de la ciudad, tanto en términos de distancia como de medios e infraestructura de transporte disponibles. Esta tendencia incluso se ha acentuado en los nuevos barrios de vivienda social construidos masivamente desde inicios de los noventa y en donde vive un porcentaje bastante significativo de la población de escasos recursos de la ciudad (Hidalgo, 2007; Tironi, 2003). Dada esta situación la posesión de un automóvil es vista como la forma privilegiada en la cual se puede vencer las limitaciones de estas locaciones y acceder de manera más fácil a una amplia gama de destinos urbanos.

La relevancia de la automovilidad como forma de hacer accesible sectores periféricos se ve potenciada por la política de construcción de infraestructura vial que se ha seguido en la ciudad en torno al modelo de las carreteras urbanas concesionadas. Esto se hace evidente cuando analizamos el caso de Fernanda, que vive en la villa Isabel Riquelme de Maipú. Esta villa se encuentra ubicada casi en el límite de la zona urbana de la ciudad, a un costado de la Autopista del Sol. Como resultado de esta situación existe una gran diferencia en términos de tiempo entre tomar la autopista y tomar transporte público (que se desplaza por calles interiores de la comuna) para llegar a las zonas centrales de la ciudad, como ella reconoce:

- ¿Y el auto es importante para ustedes?

Sí, ahora sí, con el transporte que hay ahora yo pienso que es muy importante el auto porque no podría andar en micro yo, no y si hay que salir no salgo... se demoran igual harto, una hora y media, dos horas y uno en auto se demora veinte minutos... Sí po’ uno sale a la carretera del sol pa'l centro y en un ratito, en veinte minutos estás en el centro.

- ¿Y ustedes ocupan mucho la carretera?

Sí, mucho, mucho, $p a^{\prime}$ todos lados... si vamos donde mi hermano vamos por la carretera el sol, si vamos $p a$ ' donde mi suegra vamos por la carretera el sol, no nos vamos por entre medio porque ella vive en el quince de Pajaritos entonces bajamos un poquito por Vespucio más o menos y ella vive por ahí cerca... ocupamos mucho la carretera del sol (Fernanda, 30 años, Maipú).

En segundo lugar, la relevancia de la accesibilidad también se puede analizar desde una perspectiva del riesgo. Como identifica Mariela, lo importante no es utilizar el automóvil en cualquier situación, sino tenerlo disponible cuando haya alguna emergencia que lo amerite. En los casos bajo estudio este elemento de riesgo se conecta con la automovilidad de dos formas principales. Por un lado, como lo hace Mariela, la percepción de un riesgo probable hace parecer como necesaria la forma de movilidad autónoma asociada al automóvil para llegar rápidamente a lugares como hospitales y comisarías. Por el otro, comúnmente las calles y el transporte público son identificados como áreas potencialmente peligrosas ante las cuales la imagen de seguridad 
y privacidad que ofrece al automóvil es ampliamente valorada. Henderson (2006) llama a este proceso "automovilidad secesionista", en el sentido de que el automóvil es utilizado como un medio para aislarse de un contexto urbano que es percibido como potencialmente peligroso y/o dañino. Especialmente en los casos de familias con niños pequeños el uso del automóvil "puede dar a los padres un sentimiento de empoderamiento en un contexto de inseguridad generalizada” (Sheller y Urry, 2000, p. 230).

Este último aspecto, la presencia de niños en el hogar, también es mencionado con relativa frecuencia cuando se habla de la motivación para comprar un automóvil. Por ejemplo Jonathan nos decía lo siguiente:

-Cuénteme por qué le gustaría tener auto.

Por lo niños $p o^{\prime} .$. lo que pasa es que de repente nosotros, ponte tú tengo que hacer algo, no nosotros salimos cuando queramos, entonces de repente no podemos ir por el día, si tuviésemos auto por último vamos a almorzar con amigos o de repente queremos ir al [persa] Bío - Bío o no sé, ir a cualquier parte y no sé po’ tenimos que tomar auto, porque no tenemos auto, Jorge de repente va a fiesta en la noche, la Nicole igual, o sea tengo que conseguirme auto $p a$ 'la dos o tres de la mañana irlos a buscar (Jonathan, 47 años, Recoleta).

Esta conexión entre paternidad y automovilidad es bastante recurrente. Para muchos padres el tipo de viajes que implica la paternidad, no solamente por el hecho de mover a los hijos sino también toda la serie de objetos anexos que los acompañan (mochilas, bolsos, coches, etc.), hace muy dificultoso utilizar transporte público. Junto con estos las diferentes rutinas de los niños, desde los viajes al colegio hasta las fiestas y otras reuniones sociales, transforman al automóvil en el medio de transporte ideal para lograr coordinarlas con las rutinas y trayectos de los mismos padres.

Como se puede observar, entonces, el automóvil y las formas de movilidad urbana que éste permite son valorados por motivos bastante heterogéneos, desde la configuración de la ciudad hasta el grado de comodidad y flexibilidad en los viajes que esta tecnología proporciona. Sin embargo hay un elemento que tiende a unificar las diferentes líneas de argumentación encontradas en nuestro estudio; el carácter funcional o mayoritariamente práctico de la gran mayoría de los argumentos dados para justificar la necesidad de tener un auto en el Santiago contemporáneo.

Contrario a interpretaciones de orden mayoritariamente culturalista, del tipo hecho por Bourdieu (1984), según las cuales los autos serían adquiridos principalmente para simbolizar la integración de estos segmentos a patrones de consumo tradicionalmente característicos de las clases medias, lo que encontramos es que el automóvil es demandado especialmente por la flexibilidad y comodidad que éste entrega, especialmente en el contexto de una ciudad que durante la última década ha invertido de manera importante en mejorar las condiciones para la automovilidad. Al afirmar esto no estamos descartando que los factores simbólicos o estatutarios no jueguen un rol en la opción de estos individuos por la compra y utilización de vehículos. Lo que queremos decir es que éstos comúnmente no aparecen de una forma tan prominente como los aspectos de índole funcional, al menos en términos discursivos. 
Más allá de casos particulares en los cuales el automóvil sí aparecía teniendo un importante valor simbólico, como el caso de Tomás citado al comienzo de esta sección, en general las evaluaciones de las personas bajo estudio tendían a seguir, con matices, la línea argumental presentada por Eduardo en el siguiente extracto:

- ¿Te gustaría tener un auto ahora?

Eh... sí, me gustaría, pero no es para mí un auto, o sea si mañana me compro un auto para mí no me cambia la vida ¿cachai? o sea yo el auto no lo veo como un objetivo, o como un logro, o como un reflejo de no sé qué, como lo ve puta la gente, lo ve como una proyección, tienen un auto y la cosa es que cumplí un sueño, no, para mí el auto es como el computador, es una herramienta ¿cachai? (Eduardo, 24 años, San Miguel).

Para Eduardo, así como para muchos otros participantes en el estudio, el auto es por sobre todas las cosas una herramienta para generar ciertas formas de movilidad urbana que aparecen como mejores que sus alternativas (especialmente en el contexto actual de la crisis de Transantiago). Esta valoración mayoritariamente funcional del uso del automóvil se debe a una multiplicidad de causas, aunque los altos costos involucrados en la compra y mantención de este ocupan un lugar predominante, como señala Froud et al., "los hogares pobres no tienen autos porque busquen status o auto-expresión a través de una conformidad con las normas sociales mayoritarias, ellos ocupan autos porque lo necesitan para llegar al trabajo" (2002, pp. 102-103).

\section{Conclusiones}

Como se señaló anteriormente, los niveles de automovilidad del segmento de la población bajo estudio son aún menores. Sin embargo, existen diversos indicios (saturación del mercado de grupos de ingresos altos y medios, mayor disponibilidad y menores precios, aumento en los ingresos, el fin de la exclusión femenina, etc.) que nos llevan a creer que en el corto plazo la participación de estos grupos en la propiedad y el uso cotidiano de automóviles va a aumentar de manera importante, conllevando varios de los efectos negativos detallados en la introducción de este artículo.

Desechando la alternativa simplista y socialmente discriminadora de limitar el acceso únicamente mediante alzas en los impuestos de importación y circulación de vehículos, creemos que se debe desarrollar un acercamiento más comprensivo al problema a partir de políticas públicas de desincentivo a formas de automovilidad poco sustentables. Estas políticas no deben estar limitadas a los grupos de menores ingresos, debido a su aún baja participación en los viajes totales en automóvil en la ciudad, pero tampoco pueden excluirlos debido a su probable integración masiva a esta forma de movilidad urbana en el futuro próximo.

A partir de lo presentado en este artículo, algunos elementos que deben ser considerados son los siguientes:

- Entender que la propiedady el uso son diferentes. Como se vio aquí, muchas veces la compra no responde a una demanda preestablecida, sino más que nada a una cierta oportunidad 
y la posibilidad de acceder a financiamiento. Por otro lado, en el uso diario del vehículo entran a jugar en mayor grado elementos de racionalidad económica y evaluación debido al alto costo implicado en mantener al auto en funcionamiento. En este sentido se podría pensar en políticas que no se concentraran en desincentivar la compra (la cual tiene un claro componente simbólico y es difícil que disminuya ceteris paribus) sino más que nada en ciertas prácticas de uso concretas.

- Luchar contra el establecimiento de hábitos de automovilidad, especialmente en términos de viajes al trabajo. Como ocurre con muchas otras tecnologías, al llegar al hogar el automóvil es un objeto sin funciones claramente establecidas, las cuales solo van a ir decantando con el paso del tiempo. En este proceso se debiera buscar incentivar ciertos usos del automóvil (en fines de semana, en zonas periféricas) y desincentivar otros (especialmente viajes al trabajo en horas punta), porque una vez que éstos se convierten en hábito van a ser muy difíciles de cambiar.

- Observar la automovilidad como parte de un gran sistema sociotécnico. Un elemento clave al diseñar campañas efectivas en esta área es entender que la propiedad y el uso de automóviles por parte de las personas no se dan en el vacío, sino que forman parte de un sistema sociotécnico mayor que involucra un infinidad de elementos, desde las campañas de marketing de los productores de automóviles hasta la política de infraestructura urbana del Ministerio de Obras Públicas.

- Socializar los reales costos del automóvil, tanto personales como sociales y ambientales. Entre los participantes de este estudio tendía a primar la idea de que el único costo del automóvil era monetario. En esta situación es fundamental que la política sea efectiva en socializar los reales costos del automóvil, tanto en términos personales (quizás enfatizando los usos alternativos que se podrían dar al dinero destinado a la mantención de este) como sociales y medioambientales.

- Derribar "mitos" de la automovilidad. Junto con lo anterior es fundamental que la campaña sea efectiva en atacar los mitos que subyacen a la cultura de la automovilidad contemporánea (el auto como sinónimo de libertad, autonomía, poder, estilo, etc.) y que explican en alto grado por qué se ha convertido en la forma de movilidad dominante en nuestras sociedades.

- Introducir una perspectiva de género. Como se vio, las relaciones con la automovilidad son muy diferentes entre hombres y mujeres. Por este motivo una política efectiva tendrá que diseñar estrategias específicas y focalizadas para cada uno de ellos.

Obviamente estas políticas deben ir de la mano con la provisión de infraestructuras e incentivos a formas de movilidad urbana sustentable como caminatas o bicicletas y, especialmente, una mejora considerable en la calidad del transporte público en la ciudad, dejando atrás los problemas enfrentados en la implementación del Transantiago.

Sin embargo no se puede concluir que un transporte público de calidad automáticamente va a solucionar los problemas derivados de las formas de movilidad urbana asociadas al creciente uso de automóviles por parte de grupos de menores ingresos. Hacer esto sería reducir la opción por la automovilidad solamente a un producto de una cierta racionalidad económica por parte de los miembros de estos grupos, la cual va a ser fácilmente alterada cuando los beneficios (principalmente económicos) asociados al uso de transporte público sean percibidos 
como mayores que los del uso de automóviles. Como ha ocurrido en diversas ciudades a nivel mundial, incluso en aquellas con sistemas de transporte público de niveles óptimos, la disponibilidad de alternativas económicamente más baratas y eficientes no afecta necesariamente los patrones de automovilidad de la población.

Desde el punto de vista desarrollado en este artículo esto se debe a que el automóvil es mucho más que un simple medio de transporte. El acto de adquirir y conducir uno es también un acto político; una serie de prácticas y formas discursivas que van mucho más allá del moverse de un lado al otro de la ciudad de la manera más rápida o confortable posible. Cada vez que las personas encienden el motor de un automóvil, que lo lavan los domingos, que lo defienden en una conversación o sueñan con comprarse uno están haciendo también una cierta declaración de principios acerca del tipo de ciudad y el tipo de sociedad en la cual quieren vivir. Sólo si se deja de lado la perspectiva reduccionista de que la automovilidad es un ámbito de injerencia meramente técnico o económico y se toman los elementos políticos, sociales y culturales de ésta en consideración podremos diseñar políticas que efectivamente nos ayuden a incentivar mayores niveles de movilidad sustentable en el Santiago actual.

\section{Referencias bibliográficas}

Bohm, S.; Jones, C.; Land, C. \& Paterson, J. (Eds.) (2006). Against automobility. Oxford: Blackwell Publishing.

Bourdieu, P. (1984). Distinction: a social critique of the judgment of taste. London: Routledge.

Carrabine, E. \& Longhurst, B. (2002). Consuming the car: anticipation, use and meaning in contemporary youth culture. The sociological review, 50, 2, 181-196.

Cass, N.; Shove, E. \& Urry, J. (2005). Social exclusion, mobility and access. The Sociological Review, 53, 3, 539-555.

Cresswell, T. (2006). On the move: mobility in the modern western world. London: Routledge.

Crowley, J. (1999). The sensibility of comfort. The American Historical Review, 104, 3, $1-43$.

Dant, T. \& Martin P. (2001). By car: Carrying modern society. En A. Warde \& J. Grunow (Eds.), Ordinary Consumption. London: Routledge.

De Mattos, C.; Ducci, M. E.; RodrÍguez, A. \& Yáñez, G. (Eds.) (2004). Santiago en la globalización, ¿una nueva ciudad? Santiago, Chile: SUR-EURE.

De Mattos, C. \& Hidalgo, R. (Eds.). (2007). Santiago de Chile. Movilidad espacial y reconfiguración metropolitana. Santiago: Eure Libros - Geolibros.

Ducci, M. E. (2002). Área urbana de Santiago 1991-2000: expansión de la industria y la vivienda. Eure, 28, 85, 187-207.

European Commission (2007). Green paper: Towards a new culture of urban mobility. Brussels: Commission of the European Communities.

Featherstone, M.; Thrift, N. \& Urry, J. (Eds.) (2005). Automobilities. London: Sage.

Froud, J.; Johal, S.; Leaver, A. \& Williams, K. (2002). Not enough money: The resources and choices of the motoring poor. Competition \& Change, 6, 1, 95-111. 
Galetovic, A. (Ed.) (2006). Santiago: dónde estamos y hacia dónde vamos. Santiago, Chile: Centro de Estudios Públicos.

Gartman, D. (2004). Three ages of the automobile. The cultural logics of the car. Theory, Culture \& Society, 21, 4-5, 169-195.

Goldman, T. \& Gorham, R. (2006). Sustainable urban transport: four innovative directions. Technology in Society, 28, 1-2, 261-273.

Graham, S. \& Marvin, S. (2001). Splintering ubanism. Networked infrastructures, technological mobilities and the urban condition. London and New york: Routledge.

Greene, M. \& Mora, R. (2005). Las autopistas urbanas concesionadas: una nueva forma de segregación. $A R Q, 60,56-58$.

Hagman, O. (2006). Morning queues and parking problems. On the broken promises of the automobile. Mobilities, 1, 1, 63-74.

Henderson, J. (2006). Secessionist automobility: Racism, anti-urbanism, and the politics of automobility in Atlanta, Georgia. International Journal of Urban and Regional Research, 30, 2, 293-307.

Hidalgo, R. (2007). ¿Se acabó el suelo en la gran ciudad? Las nuevas periferias metropolitanas de la vivienda social en Santiago de Chile. EURE, 23, 98, 57-75.

Instituto Nacional de Estadísticas, INE (2003). Nace un Nuevo Chile. Resultados del Censo 2002. Santiago: INE y Consejo Nacional de la Cultura y las Artes.

Instituto Nacional de Estadísticas, INE (2008). Parque de Vehiculos en Circulación, 2007. Santiago, Chile: INE.

Jiron, P. (2007). Unravelling invisible inequalities in the city through urban daily mobility. The case of Santiago, Chile. Swiss Journal of Sociology, 33,1, 45-68.

Kemp, R.. \& Rotmans, J. (2005). The management of the co-evolution of technical, environmental and social systems. En H. Weberm (Ed.), Towards environmental innovation systems. Berlin: Springer.

Kenyon, S.; Rafferty, J. \& Lyons, G. (2002). Transport and social exclusion: investigating the possibility of promoting inclusion through virtual mobility. Journal of Transport Geography, 10, 3, 207-219.

Lanfranco, P. (Ed.) (2003). Muévete por tu ciudad: una propuesta ciudadana de transporte con equidad. Santiago, Chile: LOM y Ciudad Viva.

Larrañaga, O. (2004). Población vulnerable, vivienda, patrimonio y vecindario. En J. Ramos et al. (Eds.), Cómo ha cambiado la vida de los chilenos. Análisis comparativo de las condiciones de vida de los hogares con menor bienestar económico (Censos 1992 y 2002). Santiago, Chile: INE publicaciones.

Miller, D. (Ed.) (2001). Car cultures. Oxford: Berg.

Ministerio de Vivienda y Urbanismo, MINVU (2007). Diagnóstico urbano 1990-2006. Santiago, Chile: MINVU, Gobierno de Chile.

Nowotny, H. (1994). Time. The modern and postmodern experience. Cambridge: Polity Press.

Ramos, J.; Valenzuela, E.; Larrañaga, O.; Medrano, P. \& Gubbins, V. (Eds.) (2004). Cómo ha cambiado la vida de los chilenos. Análisis comparativo de las condiciones de vida en los hogares con menor bienestar económico (Censos 1992-2002). Santiago, Chile: INE, Gobierno de Chile. 
Secretaría de Transportes, SECTRA (2002). Encuesta origen destino de viajes, Santiago 2001Informe de difusión. Santiago, Chile: SECTRA, Gobierno de Chile.

Sheller, M. (2004). Automotive emotions: feeling the car. Theory, culture \& society, 21, 4-5, 221-242.

Sheller, M. \& Urry, J. (2000). The city and the car. International Journal of Urban and Regional Research, 24, 4, 737-757.

Steg, L. \& Gifford, R. (2005). Sustainable transportation and quality of life. Journal of Transport Geography, 13, 1, 59-69.

Taylor, N. (2003). The aesthetic experience of traffic in the modern city. Urban Studies, 40, $8,1609-1625$.

Tironi, M. (2003). Nueva pobreza urbana, vivienda y capital social: análisis comparado 1985 2001. Santiago, Chile: RIL-PREDES.

Ureta, S. (2006). Machines for living in. Communication technologies and everyday life in times of urban transformation - PhD Thesis. London: London School of Economics.

Ureta, S. (2008). To move or not to move? Social exclusion, accessibility and daily mobility among the low-income population in Santiago, Chile. Mobilities, 3, 2, 269-289.

Urry, J. (2000). Sociology beyond societies: Mobilities for the twenty-first century. London: Routledge.

Urry, J. (2004). The 'system' of automobility. Theory, Culture \& Society, 21, 4/5, 25-39.

Urry, J. (2007). Mobilities. Cambridge: Polity Press.

Volti, R. (2008). A car for the great Asian multitude. Technology and Culture, 49, 4, $995-$ 1001.

Walsch, M. (2008). Gendering mobility: women, work and automobility in the United States. History, 93, 311, 376-395.

Wright, C. \& Curtis, B. (2005). Reshaping the motor car. Transport Policy, 12, 1, 11-22. 